\title{
A phase Ib/ll study of xentuzumab, an IGF-neutralising antibody, combined with exemestane and everolimus in hormone receptor-positive, HER2-negative locally advanced/metastatic breast cancer
}

Peter Schmid ${ }^{1 *}$, Marie-Paule Sablin², Jonas Bergh ${ }^{3}$, Seock-Ah $\mathrm{Im}^{4}$, Yen-Shen Lu ${ }^{5}$, Noelia Martínez ${ }^{6}$, Patrick Neven ${ }^{7}$, Keun Seok Lee ${ }^{8}$, Serafín Morales ${ }^{9}$, J. Alejandro Pérez-Fidalgo ${ }^{10}$, Douglas Adamson ${ }^{11}$, Anthony Gonçalves ${ }^{12}$, Aleix Prat ${ }^{13}$, Guy Jerusalem ${ }^{14}$, Laura Schlieker ${ }^{15}$, Rosa-Maria Espadero ${ }^{16}$, Thomas Bogenrieder ${ }^{17,18}$, Dennis Chin-Lun Huang ${ }^{19,20}$, John Crown $^{21 \dagger}$ and Javier Cortés $22,23 \dagger$

\begin{abstract}
Background: Xentuzumab—a humanised IgG1 monoclonal antibody — binds IGF-1 and IGF-2, inhibiting their growth-promoting signalling and suppressing AKT activation by everolimus. This phase Ib/II exploratory trial evaluated xentuzumab plus everolimus and exemestane in hormone receptor-positive, locally advanced and/or metastatic breast cancer (LA/MBC).

Methods: Patients with hormone receptor-positive/HER2-negative LA/MBC resistant to non-steroidal aromatase inhibitors were enrolled. Maximum tolerated dose (MTD) and recommended phase II dose (RP2D) of xentuzumab/ everolimus/exemestane were determined in phase I (single-arm, dose-escalation). In phase II (open-label), patients were randomised 1:1 to the RP2D of xentuzumab/everolimus/exemestane or everolimus/exemestane alone. Randomisation was stratified by the presence of visceral metastases. Primary endpoint was progression-free survival (PFS).

(Continued on next page)
\end{abstract}

\footnotetext{
* Correspondence: p.schmid@qmul.ac.uk

†John Crown and Javier Cortés contributed equally to this work.

${ }^{1}$ Centre for Experimental Cancer Medicine, Barts Cancer Institute, Queen

Mary University of London, London, UK

Full list of author information is available at the end of the article
}

(c) The Author(s). 2021 Open Access This article is licensed under a Creative Commons Attribution 4.0 International License, which permits use, sharing, adaptation, distribution and reproduction in any medium or format, as long as you give appropriate credit to the original author(s) and the source, provide a link to the Creative Commons licence, and indicate if changes were made. The images or other third party material in this article are included in the article's Creative Commons licence, unless indicated otherwise in a credit line to the material. If material is not included in the article's Creative Commons licence and your intended use is not permitted by statutory regulation or exceeds the permitted use, you will need to obtain permission directly from the copyright holder. To view a copy of this licence, visit http://creativecommons.org/licenses/by/4.0/ The Creative Commons Public Domain Dedication waiver (http://creativecommons.org/publicdomain/zero/1.0/) applies to the data made available in this article, unless otherwise stated in a credit line to the data. 
(Continued from previous page)

Results: MTD was determined as xentuzumab 1000 mg weekly plus everolimus 10 mg/day and exemestane 25 mg/day. A total of 140 patients were enrolled in phase II (70 to each arm). Further recruitment was stopped following an unfavourable benefit-risk assessment by the internal Data Monitoring Committee appointed by the sponsor. Xentuzumab was discontinued; patients could receive everolimus/exemestane if clinically indicated. Median PFS was 7.3 months (95\% Cl 3.3-not calculable) in the xentuzumab/everolimus/exemestane group and 5.6 months (3.7-9.1) in the everolimus/exemestane group (hazard ratio $0.97,95 \% \mathrm{Cl} 0.57-1.65 ; P=0.9057$ ). In a pre-specified subgroup of patients without visceral metastases at screening, xentuzumab/everolimus/exemestane showed evidence of PFS benefit versus everolimus/exemestane (hazard ratio 0.21 [0.05-0.98]; $P=0.0293$ ). Most common any-cause adverse events in phase II were diarrhoea (29 [41.4\%] in the xentuzumab/everolimus/exemestane group versus 20 [29.0\%] in the everolimus/exemestane group), mucosal inflammation (27 [38.6\%] versus 21 [30.4\%]), stomatitis (24 [34.3\%] versus 24 [34.8\%]), and asthenia (21 [30.0\%] versus 24 [34.8\%]).

Conclusions: Addition of xentuzumab to everolimus/exemestane did not improve PFS in the overall population, leading to early discontinuation of the trial. Evidence of PFS benefit was observed in patients without visceral metastases when treated with xentuzumab/everolimus/exemestane, leading to initiation of the phase II XENERA ${ }^{\mathrm{TM}}$-1 trial (NCT03659136).

Trial registration: ClinicalTrials.gov, NCT02123823. Prospectively registered, 8 March 2013.

Keywords: Breast cancer, HER2-negative, Hormone receptor-positive, Insulin-like growth factor, Xentuzumab

\section{Introduction}

Standard treatment for postmenopausal women with hormone receptor-positive, human epidermal growth factor receptor (HER2)-negative advanced breast cancer is endocrine therapy, using an aromatase inhibitor (AI; e.g. letrozole, anastrozole [non-steroidal], or exemestane [steroidal]), tamoxifen, or fulvestrant [1]. Despite initial benefit, disease progression typically ensues due to acquired endocrine resistance. Additionally, patients may have primary resistance, rendering them unresponsive to endocrine therapy [2].

Strategies to prevent acquired endocrine resistance include inhibition of cell-cycle progression in breast cancer cells using cyclin-dependent kinase (CDK) inhibitors [2]. The addition of a CDK 4/6 inhibitor (e.g. palbociclib, ribociclib, and abemaciclib) to an AI in endocrine therapy-naïve or endocrine-pre-treated patients, or to fulvestrant in endocrine-pre-treated patients, improved progression-free survival (PFS) and overall survival (OS) $[3,4]$ and is recommended in current treatment guidelines [1].

Alternatively, treatment or reversal of endocrine resistance may be achieved by targeting molecular pathways that become activated during acquired resistance [2]. While activating ESR1 mutations have emerged as a key mechanism in resistance to AIs (but not to fulvestrant) [5], adaptive signalling via the mammalian target of rapamycin (mTOR)/phosphoinositide 3-kinase (PI3K)/ protein kinase B (AKT) pathway also plays an important role [2]. Everolimus, an mTOR inhibitor, is approved in combination with exemestane for advanced breast cancer with endocrine resistance, having demonstrated improved PFS versus exemestane alone in the BOLERO-2 trial $[6,7]$; however, in contrast to some CDK4/6 inhibitors $[3,4]$, OS benefit was not observed. Additionally, everolimus was associated with a higher incidence of adverse events (AEs), such as stomatitis, fatigue, and hyperglycaemia. Notably, everolimus plus exemestane has shown similar efficacy irrespective of prior CDK4/6 inhibitor therapy [8].

Insulin-like growth factor (IGF) signalling can influence cancer progression and prognosis, and drives resistance to various anti-cancer treatments [9]. In breast cancer, regulatory feedback loops between the IGF axis and the mTOR/PI3K/AKT pathway may limit the efficacy of mTOR inhibitor/endocrine therapy combinations due to compensatory IGF liganddriven signalling [2, 10]. Preclinical data also suggest that IGF signalling may have a key role in nonvisceral disease, particularly bone and lymph node metastases development [11, 12].

Xentuzumab (BI 836845), a humanised IgG1 monoclonal antibody, binds IGF-1 and IGF-2 ligands with high affinity and potently neutralises their proliferative signalling [10]. Xentuzumab has shown preclinical activity across a range of cancer types [10]. Xentuzumab monotherapy was associated with mild-to-moderate AEs, most commonly gastrointestinal disorders, and preliminary anti-tumour activity in two phase I studies [13]. We hypothesised that combining xentuzumab with everolimus and exemestane would block the negative feedback between IGF and PI3K/AKT/mTOR signalling, thus enhancing sensitivity and/or overcoming endocrine resistance. This phase I/II study (NCT02123823) evaluated the efficacy and safety of xentuzumab plus everolimus and exemestane, versus exemestane and everolimus 
alone, in women with locally advanced or metastatic breast cancer $(\mathrm{MBC})$.

\section{Materials and methods Study design}

This study comprised a single-arm, dose-escalation phase I part to determine the maximum tolerated dose (MTD) and recommended phase II dose (RP2D) of xentuzumab in combination with everolimus and exemestane, and a two-arm, open-label, randomised, parallel-design phase II part to evaluate the anti-tumour activity of this triple combination versus everolimus and exemestane.

The phase I part included a 7-day run-in with everolimus and exemestane to achieve steady state prior to xentuzumab treatment. Dose escalation proceeded using a $3+3$ design. The starting dose was everolimus $10 \mathrm{mg}$ orally once daily (QD) and xentuzumab $750 \mathrm{mg}$ intravenously once weekly. All cohorts received oral exemestane $25 \mathrm{mg}$ QD. In the open-label phase II part, patients were randomised (1:1), stratified by visceral involvement (yes/no; where visceral refers to lung, liver, brain, pleural, and peritoneal metastasis), to everolimus $10 \mathrm{mg}$ QD plus exemestane $25 \mathrm{mg}$ QD, or xentuzumab plus everolimus (at the RP2D determined in phase I) and exemestane $25 \mathrm{mg}$ QD. Proactive management of stomatitis was mandated, including use of mouthwash. Treatment continued in 28-day cycles until progression, intolerable AEs, or other reasons.

The study was approved by the Institutional Review Board or Independent Ethics Committee, respectively, with jurisdiction for the participating sites and was performed in accordance with the Declaration of Helsinki and International Conference on Harmonisation Good Clinical Practice. All patients received oral and written study information and provided written, informed consent. In the phase II part, an internal data monitoring committee (DMC) - comprised of an independent group of experts from the sponsor-was appointed to assess the benefit-risk balance of xentuzumab in combination with everolimus and exemestane (two planned timepoints: after approximately 30 and 45 PFS events). Following the first analysis, the DMC advised termination of the study based on a claimed negative benefitrisk balance. Patient recruitment was stopped on 28 October 2016, and xentuzumab was discontinued; however, patients could continue to receive everolimus and exemestane if clinically indicated.

\section{Patients}

Full criteria are provided in Supplementary methods (see Additional file 1). Briefly, eligible patients were postmenopausal women aged $\geq 18$ years, with histologically confirmed, locally advanced or MBC that was not amenable to curative therapy, and had recurred or progressed on/ after the last line of systemic therapy for breast cancer. Patients were required to have hormone receptorpositive, HER2-negative tumours per local testing, and adequate archival tumour tissue. Patients had tumours that were resistant to non-steroidal AIs (defined as recurrence during/within 12 months after adjuvant treatment or progression during/within 1 month after treatment for locally advanced or MBC). Additional inclusion criteria included the following: life expectancy of $\geq 6$ months, adequate organ function, a measurable lesion per Response Evaluation Criteria in Solid Tumors (RECIST) v.1.1 or bone lesions (lytic or mixed) in the absence of a measurable lesion, and Eastern Cooperative Oncology Group performance status $\leq 2$. Exclusion criteria included the following: previous treatment with agents targeting the IGF, PI3K, AKT, or mTOR pathways; prior exemestane treatment; brain or central nervous system metastases; and $>2$ previous lines of chemotherapy for locally advanced/MBC ( $>1$ previous line in phase II). There was no limit on lines of prior endocrine therapies, and prior CDK4/6 inhibitor treatment was permitted.

\section{Endpoints and assessments}

The primary phase I endpoint was MTD, defined as the highest dose at which no more than one of six MTDevaluable patients experienced a dose-limiting toxicity (DLT; Supplementary Table 1, Additional File 1) during the first treatment cycle (MTD evaluation period). The primary phase II endpoint was PFS per investigator review (time from the date of randomisation until the date of the first progression according to RECIST v.1.1, or death). Secondary endpoints (phase II) were time to progression (TTP), objective response (OR; complete response [CR] or partial response [PR] per RECIST v.1.1), time to OR, duration of OR, disease control (CR or PR, or stable disease or non-CR/non-progressive disease lasting $\geq 24$ weeks), and duration of disease control. Further phase II endpoints included OS (duration from date of randomisation to the date of death). AEs were graded per the National Cancer Institute Common Terminology Criteria for Adverse Events (NCI CTCAE) v.4.03.

\section{Statistical analysis}

In phase II, sample size was calculated based on a hazard ratio (HR) of 0.72 , equating to a median PFS increase from 7.8 months for everolimus/exemestane to 10.8 months for the triple combination. It was calculated that 90 PFS events were required, with an estimated study duration of around 30 months. Based on these considerations, 150 patients were planned to be randomised. Following the DMC recommendation to terminate the study, recruitment was stopped and xentuzumab 
Table 1 Baseline characteristics: phase II part

\begin{tabular}{|c|c|c|c|}
\hline & $\mathrm{Xe} 1000+\operatorname{Ev} 10+\operatorname{Ex} 25(n=70)$ & $\operatorname{Ev} 10+\operatorname{Ex} 25(n=70)$ & Total $(n=140)$ \\
\hline Median age, years (range) & $58.5(41-86)$ & $62.0(42-84)$ & $60.0(41-86)$ \\
\hline \multicolumn{4}{|l|}{ ECOG PS, $n(\%)$} \\
\hline 0 & $45(64.3)$ & $38(54.3)$ & $83(59.5)$ \\
\hline 1 & $25(35.7)$ & $31(44.3)$ & $56(40.0)$ \\
\hline 2 & 0 & $1(1.4)$ & $1(0.7)$ \\
\hline Median time since diagnosis, months (range) & $63.4(12.4-343.5)$ & $63.3(8.3-256.6)$ & $63.3(8.3-343.5)$ \\
\hline \multicolumn{4}{|l|}{ Metastatic sites at screening, $n$ (\%) } \\
\hline 1 & $18(25.7)$ & $16(22.9)$ & $34(24.3)$ \\
\hline 2 & $25(35.7)$ & $27(38.6)$ & $52(37.1)$ \\
\hline$\geq 3$ & 27 (38.6) & $27(38.6)$ & $54(38.6)$ \\
\hline \multicolumn{4}{|l|}{ Visceral involvement, $n(\%)$} \\
\hline Yes & $53(75.7)$ & $54(77.1)$ & $107(76.4)$ \\
\hline No & $17(24.3)$ & $16(22.9)$ & $33(23.6)$ \\
\hline Bone metastases, $n(\%)$ & $47(67.1)$ & $52(74.3)$ & $99(70.7)$ \\
\hline Lymph node metastases, $n$ (\%) & $33(47.1)$ & $25(35.7)$ & $58(41.4)$ \\
\hline Prior hormone therapy, $n(\%)$ & $68(97.1)$ & $69(98.6)$ & $137(97.9)$ \\
\hline Prior chemotherapy in metastatic setting, $n(\%)$ & $17(24.3)$ & $18(25.7)$ & $35(25.0)$ \\
\hline Prior CDK 4/6 inhibitor treatment, $n(\%)$ & 0 & $2(2.9)$ & $2(1.4)$ \\
\hline
\end{tabular}

CDK cyclin-dependent kinase, ECOG PS Eastern Cooperative Oncology Group performance status

treatment discontinued. To allow a meaningful treatment group comparison, analysis of all RECIST endpoints was based on a data cut-off of 25 November 2016. Mandatory collection of OS data was also stopped after a protocol amendment.

No formal statistical testing was planned; all analyses were exploratory. Efficacy analysis (phase II) included all randomised patients; safety analyses included all treated patients. A Cox proportional hazards model stratified by visceral involvement (yes/no) was used to estimate the HR for PFS between treatment arms. Median PFS was calculated from Kaplan-Meier curves with 95\% confidence intervals (CIs) using the Greenwood variance estimate. Exploratory $P$ values were calculated by two-sided log-rank test. OR rates (ORRs) and disease control rates (DCRs) were compared based on the odds ratio resulting from logistic regression, adjusted for visceral involvement. Exploratory $P$ values were calculated using the likelihood ratio test. Pre-specified subgroup analyses for PFS included visceral involvement, bone-only metastasis, age, and measurable disease at baseline. Median duration of follow-up was calculated via the reverse Kaplan-Meier method.

\section{Results}

Phase I

Patients and treatment

Twenty-four patients were treated in phase I (xentuzumab $750 \mathrm{mg}$ + everolimus $10 \mathrm{mg}$ + exemestane $25 \mathrm{mg}$ [Xe750+Ev10+Ex25], $n=3$; xentuzumab $1000 \mathrm{mg}+$ everolimus $10 \mathrm{mg}+$ exemestane $25 \mathrm{mg}$ [Xe1000+Ev10+ Ex25], $n=21$; Supplementary Fig. 1 and Supplementary Table 2, Additional File 1).

\section{MTD and DLTS}

During the MTD evaluation period, 0/3 patients in the Xe750+Ev10+Ex25 group and $1 / 6$ patients in the Xe1000+Ev10+Ex25 group had a DLT (grade 3 stomatitis). MTD was thus determined as Xe1000+Ev10+Ex25.

\section{Safety}

The overall safety profile in phase I is shown in Supplementary Table 3 (Additional File 1). The most common any-grade AEs were anaemia (66.7\%), decreased appetite (58.3\%), hyperglycaemia (58.3\%), and mucosal inflammation (54.2\%).

\section{Phase II \\ Patients and treatment}

In the phase II part, 140 patients were randomised (70 to each treatment), and 139 were treated (one patient in the Ev10+Ex25 arm died and was not treated; Supplementary Fig. 1, Additional File 1). Baseline characteristics were generally well balanced between the two treatment arms (Table 1). Median (range) duration of any study treatment was $3.7(0.3-30.0)$ months in the $\mathrm{Xe} 1000+\operatorname{Ev} 10+\mathrm{Ex} 25$ arm and 5.5 (0.6-32.3) months in the Ev10+Ex25 arm. 
a

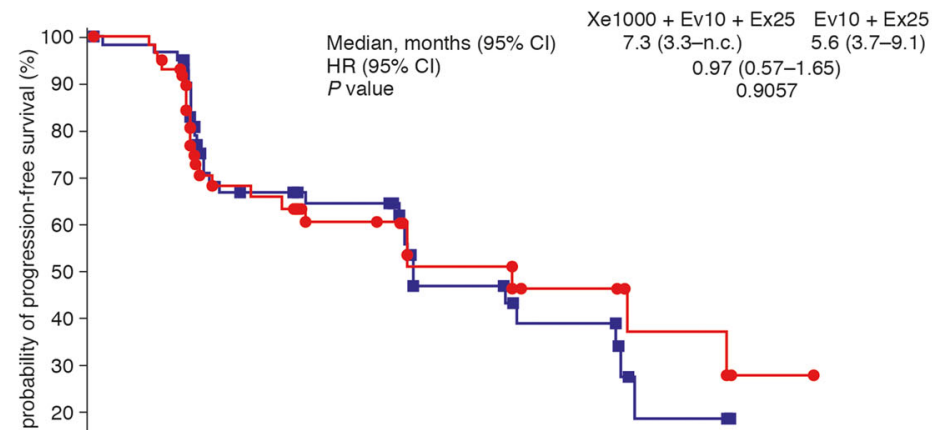

$10-\mathrm{Xe1000}+\mathrm{Ev} 10+\mathrm{Ex} 25$

- Ev10+Ex25

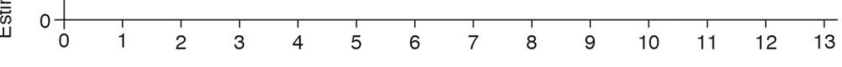

Number of patients at risk:

Time (months)

$\mathrm{Xe} 1000+\mathrm{Ev} 10+\mathrm{Ex} 25$ Ev10 + Ex25

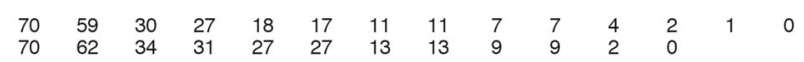

b

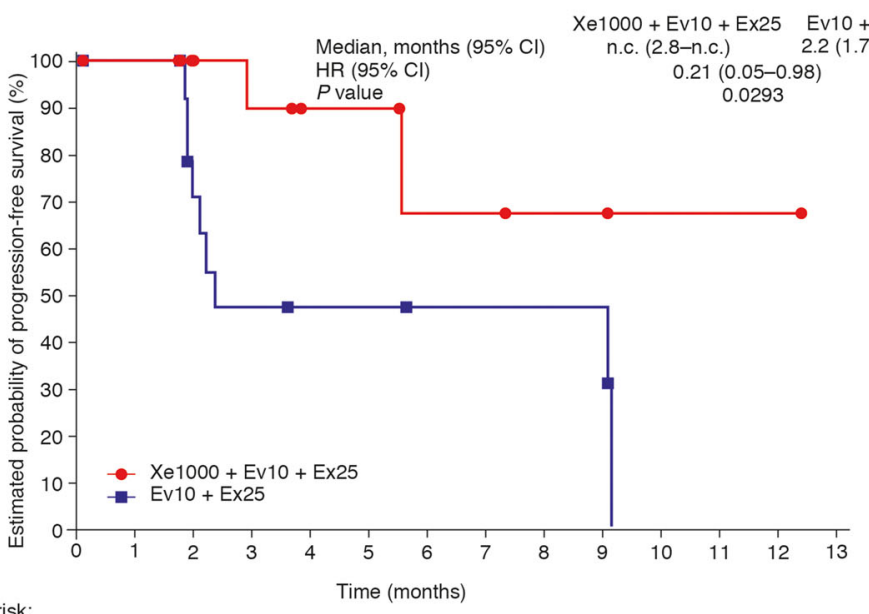

Number of patients at risk: $\mathrm{Xe} 1000+\mathrm{Ev} 10+\mathrm{Ex} 25$ Ev10 + Ex25

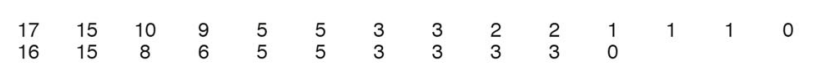

C

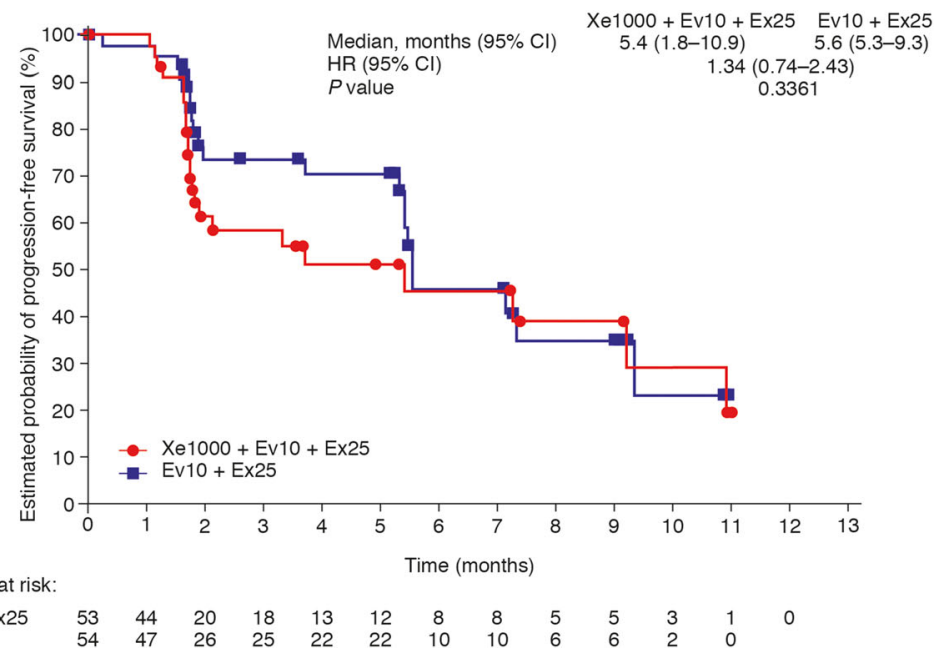

Fig. 1 PFS in a all patients, b patients without visceral involvement, and c patients with visceral involvement. Data are from phase II part. Cl, confidence interval; HR, hazard ratio; n.c., not calculable; PFS, progression-free survival 


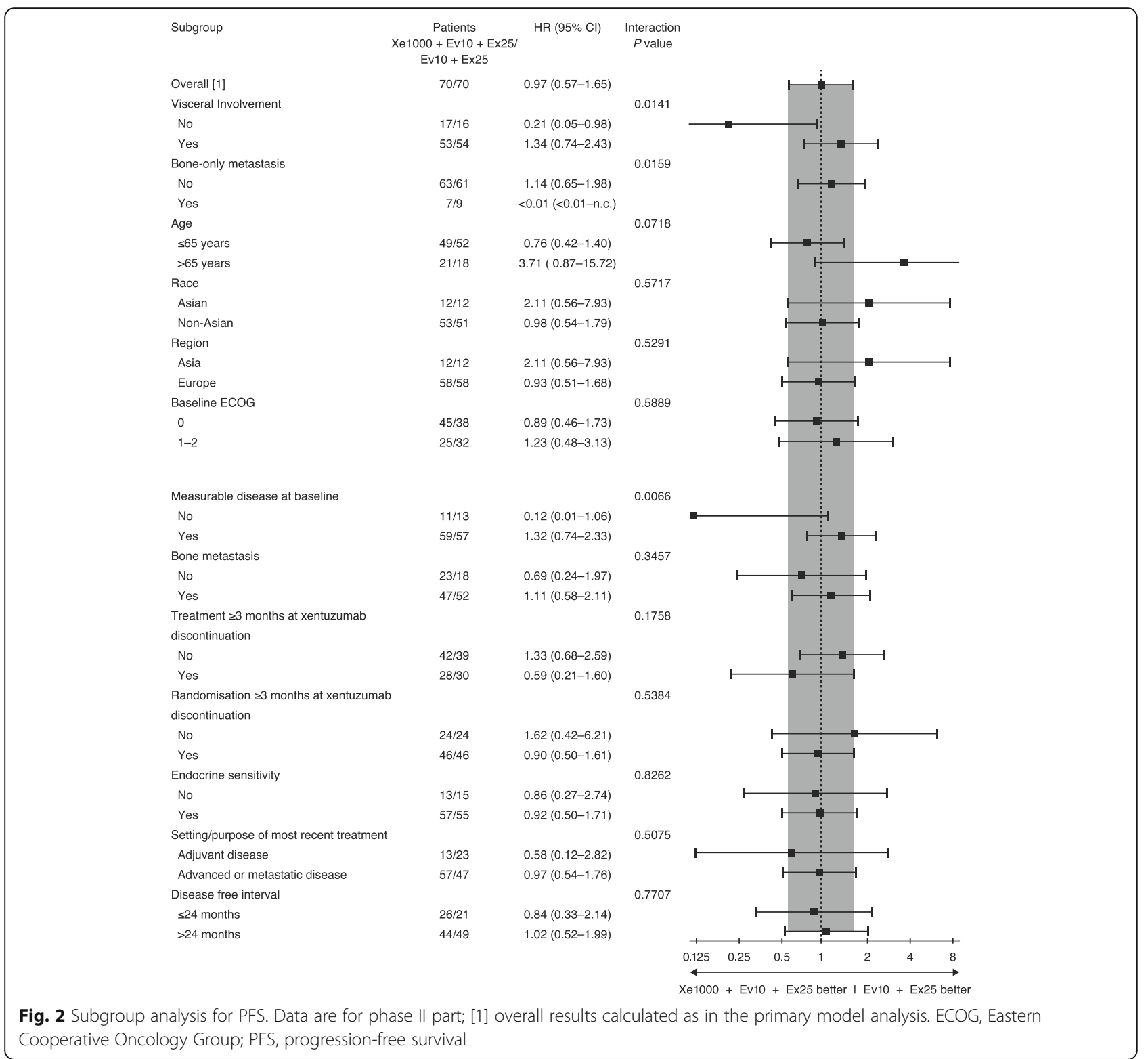

\section{Efficacy}

At the time of data cut-off for the RECIST endpoints, median duration of follow-up was 5.2 months (95\% CI 3.5-5.5).

\section{PFS}

PFS was not significantly different between the Xe1000+ Ev10+Ex25 and Ev10+Ex25 treatment arms (median [95\% CI] PFS, 7.3 months [3.3-not calculable] versus 5.6 [3.79.1]; HR 0.97 [95\% CI 0.57-1.65]; $P=0.9057$; Fig. 1a).

\section{Pre-specified subgroup analysis: visceral and non-visceral disease}

In the pre-specified subgroup of patients without visceral metastases at screening, Xe1000+Ev10+Ex25 showed evidence of PFS benefit versus Ev10+Ex25 (two PFS events in 17 patients versus nine events in 16 patients; HR 0.21 [95\% CI 0.05-0.98], $P=0.0293$ ). Median PFS in those without visceral metastases was not calculable (95\% CI 2.8-not calculable) for Xe1000+Ev10+Ex25 versus 2.2 months (95\% CI 1.7-9.1) for Ev10+Ex25 (Fig. 1b). There was no evidence of PFS benefit in patients with visceral involvement (23 PFS events in 53 patients [Xe1000+Ev10+Ex25] versus 21 events in 54 patients [Ev10+Ex25]; HR 1.34 [95\% CI 0.74-2.43], $P=$ 0.3361 ). Median PFS in those with visceral metastases at screening was 5.4 months (95\% CI 1.8-10.9) with Xe1000+Ev10+Ex25 versus 5.6 months (95\% CI 5.3-9.3) with Ev10+Ex25 (Fig. 1c). The treatment by subgroup interaction $P$ value $\left[P_{\text {int }}\right]$ was 0.0141 . Evidence of PFS 
Table 2 Best overall response: phase II part

\begin{tabular}{|c|c|c|}
\hline & Xe1000+Ev10+Ex25 $(n=70)$ & $\operatorname{Ev} 10+\operatorname{Ex} 25(n=70)$ \\
\hline \multicolumn{3}{|l|}{ Best overall response } \\
\hline$C R$ & $1(1.4)$ & 0 \\
\hline PR & $4(5.7)$ & $7(10.0)$ \\
\hline Non-CR/non-PD, $n(\%)$ & $10(14.3)$ & $8(11.4)$ \\
\hline Non-CR/non-PD $\geq 24$ weeks & $4(5.7)$ & $3(4.3)$ \\
\hline $\mathrm{SD}, n(\%)$ & $28(40.0)$ & $32(45.7)$ \\
\hline $\mathrm{SD} \geq 24$ weeks & $4(5.7)$ & $7(10.0)$ \\
\hline $\mathrm{PD}, n(\%)$ & $16(22.9)$ & $14(20.0)$ \\
\hline Not evaluable, $n(\%)$ & $11(15.7)$ & $9(12.9)$ \\
\hline $\mathrm{OR}, n(\%)$ & $5(7.1)$ & $7(10.0)$ \\
\hline Odds ratio $(95 \% \mathrm{Cl})[P \text { value }]^{\dagger}$ & \multicolumn{2}{|c|}{$0.70(0.20-2.32)[P=0.5598]$} \\
\hline Median time to OR, months (range) & $3.7(1.8-5.3)$ & $1.8(1.6-7.2)$ \\
\hline Median duration of $\mathrm{OR}_{1}^{\ddagger}$ months $(95 \% \mathrm{Cl})$ & $5.6(\mathrm{NC}-\mathrm{NC})$ & NC (1.8-NC) \\
\hline Disease control, $n(\%)$ & $13(18.6)$ & $17(24.3)$ \\
\hline Odds ratio $(95 \% \mathrm{Cl})[P \text { value }]^{+}$ & \multicolumn{2}{|c|}{$0.70(0.31-1.59)[P=0.4008]$} \\
\hline Median duration of disease control, ${ }^{\ddagger}$ months (95\% Cl) & NC $(9.2-N C)$ & $9.3(9.0-\mathrm{NC})$ \\
\hline
\end{tabular}

$C l$ confidence interval, $C R$ complete response, $N C$ not calculable, $O R$ objective response, $P D$ progressive disease, $P R$ partial response, $S D$ stable disease ${ }^{+}$Odds ratio and $P$ value are obtained from logistic regression model adjusted for visceral involvement at screening. An odds ratio $>1$ favours Xe1000+Ev10+Ex 25 ${ }^{\ddagger}$ Kaplan-Meier estimates

improvement was also observed in the nested "boneonly" metastasis subgroup (HR $<0.01[<0.01-$ not calculable]; $P=0.0942 ; P_{\text {int }}=0.0159$; Fig. 2 ).

\section{Secondary and further endpoints}

TTP was similar between treatment arms (HR 1.03 [95\% CI $0.59-1.80] ; P=0.9146)$. ORRs and DCRs were also similar between the two treatment arms (Table 2). At the time of analysis, OS data were immature and inconsistently collected. Most patients $(85.7 \%)$ had OS status censored at analysis. Overall, 20 patients had died (Xe1000+Ev10+Ex25, $n=9 ;$ Ev10+Ex25, $n=11 ;$ HR 0.79 [95\% CI 0.33-1.93]).

\section{Safety}

Following the DMC recommendation, xentuzumab treatment was discontinued; however, patients could continue to receive exemestane/everolimus. Safety analysis was therefore split according to AEs that occurred up to 9 December 2016 (i.e. the date of xentuzumab discontinuation [28 October 2016] plus the 42-day residual effect period; Table 3), and those occurring when patients were receiving only exemestane/everolimus on both treatment arms (10 December 2016 until 4 July 2018; Supplementary Table 4, Additional File 1).

Any-cause AEs reported more frequently with Xe1000+Ev10+Ex25 versus Ev10+Ex25 were abdominal pain $(14.3 \%$ versus $4.3 \%)$, diarrhoea $(41.4 \%$ versus $29.0 \%)$, headache (24.3\% versus $13.0 \%)$, muscle spasms
(11.4\% versus $1.4 \%)$, nausea (34.3\% versus $23.2 \%)$, neutropenia $(28.6 \%$ versus $15.9 \%)$, and platelet count decreased (18.6\% versus $4.3 \%)$. Infusion-related reactions with xentuzumab were rarely reported (2 [2.9\%] patients; one grade 1 and one grade 2). Selected AEs associated with everolimus were examined using grouped terms (indicated by + ). The incidence of stomatitis+ and noninfectious pneumonitis+ was similar between the two arms $(74.3 \%$ versus $72.5 \%$ and $12.9 \%$ versus $18.8 \%$, respectively). Neutropenia+ and thrombocytopenia+ were reported more frequently in the triple combination arm than the control arm $(40.0 \%$ versus $27.5 \%$ [grade $\geq 3$, $17.1 \%$ versus $7.2 \%$ ] and $40.0 \%$ versus $17.7 \%$ [grade $\geq 3$, $7.1 \%$ versus $1.4 \%]$, respectively).

Two patients in the Xe1000+Ev10+Ex25 arm died due to pneumonitis and liver injury, and two patients in the Ev10+Ex25 arm died due to progression of Burkitt's lymphoma and acute kidney injury. Except for Burkitt's lymphoma, these were considered related to treatment. The AE profile in patients without visceral involvement at screening was consistent with that in the overall trial population (data not shown).

Xentuzumab dose reduction was required in three patients in the Xe1000+Ev10+Ex25 arm. Everolimus dose reduction was required more frequently in the Xe1000+ Ev10+Ex25 arm than the Ev10+Ex25 arm (32 [45.7\%] versus 18 [26.1\%] patients). The most frequent $\mathrm{AE}$ leading to dose reduction of everolimus in the Xe1000+ Ev10+Ex25 arm was stomatitis (14 [20.0\%] patients). 
Table 3 Safety profile and most common AEs occurring up to 9 December 2016: phase II part

\begin{tabular}{|c|c|c|c|c|}
\hline Patients, $n$ (\%) & \multicolumn{2}{|c|}{ Xe1000+Ev10+Ex25 $(n=70)$} & \multicolumn{2}{|c|}{ Ev10+Ex25 $(n=69)$} \\
\hline Any $\mathrm{AE}$ & \multicolumn{2}{|l|}{$70(100.0)$} & \multicolumn{2}{|l|}{$68(98.6)$} \\
\hline Any grade $\geq 3 \mathrm{AE}$ & \multicolumn{2}{|l|}{$39(55.7)$} & \multicolumn{2}{|l|}{$31(44.9)$} \\
\hline Any TRAE & \multicolumn{2}{|l|}{$66(94.3)$} & \multicolumn{2}{|l|}{$66(95.7)$} \\
\hline Any grade $\geq 3$ TRAE & \multicolumn{2}{|l|}{$31(44.3)$} & \multicolumn{2}{|l|}{$21(30.4)$} \\
\hline Any serious $A E$ & \multicolumn{2}{|l|}{$15(21.4)$} & \multicolumn{2}{|l|}{$20(29.0)$} \\
\hline Most common any-cause AEs & Any grade & Grade $\geq 3$ & Any grade & Grade $\geq 3$ \\
\hline Diarrhoea & $29(41.4)$ & $1(1.4)$ & $20(29.0)$ & $1(1.4)$ \\
\hline Mucosal inflammation & $27(38.6)$ & $2(2.9)$ & $21(30.4)$ & 0 \\
\hline Stomatitis & $24(34.3)$ & $4(5.7)$ & $24(34.8)$ & $3(4.3)$ \\
\hline Nausea & $24(34.3)$ & 0 & $16(23.2)$ & 0 \\
\hline Rash & $23(32.9)$ & 0 & $21(30.4)$ & 0 \\
\hline Cough & $23(32.9)$ & $1(1.4)$ & $16(23.2)$ & $2(2.9)$ \\
\hline Asthenia & $21(30.0)$ & $1(1.4)$ & $24(34.8)$ & $2(2.9)$ \\
\hline Fatigue & $20(28.6)$ & 0 & $17(24.6)$ & $1(1.4)$ \\
\hline Neutropenia & $20(28.6)$ & $8(11.4)$ & $11(15.9)$ & $2(2.9)$ \\
\hline Decreased appetite & $18(25.7)$ & $1(1.4)$ & $21(30.4)$ & 0 \\
\hline Hyperglycaemia & $18(25.7)$ & $4(5.7)$ & $15(21.7)$ & $3(4.3)$ \\
\hline Headache & $17(24.3)$ & 0 & $9(13.0)$ & 0 \\
\hline Thrombocytopenia & $17(24.3)$ & $4(5.7)$ & $10(14.5)$ & $1(1.4)$ \\
\hline ALT increased & $16(22.9)$ & $3(4.3)$ & $10(14.5)$ & $1(1.4)$ \\
\hline Anaemia & $15(21.4)$ & $2(2.9)$ & $11(15.9)$ & $2(2.9)$ \\
\hline Vomiting & $14(20.0)$ & $2(2.9)$ & $13(18.8)$ & 0 \\
\hline AST increased & $13(18.6)$ & $3(4.3)$ & $13(18.8)$ & 0 \\
\hline Epistaxis & $13(18.6)$ & 0 & $9(13.0)$ & $1(1.4)$ \\
\hline Platelet count decreased & $13(18.6)$ & $1(1.4)$ & $3(4.3)$ & 0 \\
\hline Constipation & $13(18.6)$ & $1(1.4)$ & $6(8.7)$ & 0 \\
\hline Pruritus & $13(18.6)$ & 0 & $12(17.4)$ & 0 \\
\hline Nasopharyngitis & $12(17.1)$ & 0 & $6(8.7)$ & 0 \\
\hline Hypophosphataemia & $11(15.7)$ & $6(8.6)$ & $8(11.6)$ & $2(2.9)$ \\
\hline Dysgeusia & $11(15.7)$ & 0 & $10(14.5)$ & 0 \\
\hline Dyspnoea & $9(12.9)$ & $1(1.4)$ & $17(24.6)$ & $4(5.8)$ \\
\hline Peripheral oedema & $5(7.1)$ & 0 & $14(20.3)$ & 0 \\
\hline Arthralgia & $4(5.7)$ & 0 & $11(15.9)$ & 0 \\
\hline Mouth ulceration & $3(4.3)$ & 0 & $12(17.4)$ & 0 \\
\hline
\end{tabular}

$A E$ adverse event, $A L T$ alanine aminotransferase, AST aspartate aminotransferase, TRAE treatment-related adverse event. Most common AEs are those of any causality ( $\geq 15 \%$ of patients in either arm)

However, discontinuations of everolimus due to AEs were numerically lower with Xe1000+Ev10+Ex25 than Ev10+Ex25 (9 [12.9\%] versus 12 [17.4\%] patients).

\section{Discussion}

In phase I, the MTD of xentuzumab was $1000 \mathrm{mg}$ weekly in combination with everolimus $10 \mathrm{mg}$ QD and exemestane $25 \mathrm{mg}$ QD, which was also determined as the RP2D. In the randomised phase II part, we hypothesised that addition of xentuzumab to everolimus/exemestane would overcome resistance to endocrine therapy; however, at a pre-specified interim analysis on 18 October 2016, the internal DMC recommended terminating the trial due to a lack of superior efficacy in the Xe1000+Ev10+Ex25 arm. Consequently, patient recruitment was stopped early and xentuzumab was discontinued. Patients continued to receive everolimus/ 
exemestane provided they had not met discontinuation criteria.

Overall, the triple combination had a manageable and tolerable safety profile, which was remarkably similar to the safety profile of everolimus and exemestane alone. Frequency of key side effects associated with everolimus, such as pneumonitis, stomatitis/mucositis, and hyperglycaemia, was generally not increased by the addition of xentuzumab. In the phase II part, neutropenia and platelet count decreased were more common in the xentuzumab arm; however, this was not associated with increased infections.

While comparison of treatment arms was exploratory, there was no evidence of any difference in PFS between Xe1000+Ev10+Ex25 and Ev10+Ex25, and no relevant differences between treatment arms were observed regarding secondary or further efficacy endpoints. In line with the hypothesis that IGF may be important in bone and lymph node metastases, patients with non-visceral involvement were identified as a subgroup with potential benefit from treatment with Xe1000+Ev10+Ex25 versus Ev10+Ex25 (HR 0.21 [95\% CI 0.05-0.98]). There was no evidence of a treatment effect for patients with visceral disease. Evidence of PFS improvement with Xe1000+Ev10+Ex25 versus control was also observed in the "bone-only" metastasis subgroup. Some caution should be taken with drawing any firm conclusions from the subgroup analyses given the number of subgroup analyses performed and the relatively low number of patients/ events in some subgroups. Any comparisons of point estimates, such as medians, should also take into consideration corresponding 95\% CIs.

The finding of benefit in the non-visceral subgroup is interesting, particularly as in BOLERO-2, the treatment effect of Ev10+Ex25 versus Ex25 was consistent in patients with and without visceral metastases [6]. However, fulvestrant has shown improved treatment effects in patients with non-visceral disease in the FIRST and FALCON trials (fulvestrant versus anastrozole). In these trials, which included endocrine treatment-naïve postmenopausal women with advanced breast cancer, the HRs for PFS were smaller for the non-visceral subgroup than the visceral subgroup (FALCON, 0.59 [95\% CI $0.42-0.84]$ versus 0.99 [0.74-1.33]; FIRST, 0.58 [0.340.99 ] versus 0.82 [0.54-1.26]) [14]. Conversely, the PFS and OS HRs were similar in the non-visceral and visceral subgroups in CONFIRM (fulvestrant 500 versus $250 \mathrm{mg}$ in patients with prior endocrine therapy; PFS, 0.72 [0.57-0.92] versus 0.86 [0.69-1.06]; OS, 0.78 [0.61-1.01] versus 0.83 [0.67-1.04]) [14].

The IGF axis is known to contribute to bone metastases, and bone-derived IGF has been shown to mediate crosstalk between bone and metastasized cancer cells [15].
Moreover, preclinical evidence suggests that inhibition of IGF-axis signalling can attenuate the development and progression of bone metastases in breast and prostate cancers [11]. Associations of elevated expression of IGF axis components with lymph node metastases have been identified in several cancer types, including breast cancer [12, 16, 17]. Interestingly, high IGF-1R expression appears to be associated with lower metastatic potential in breast cancer [18]. This may suggest that non-visceral disease retains IGF-1R signalling, while visceral metastases lose IGF-1R expression. These results are hypothesisgenerating and warrant further examination of xentuzumab in patients with only non-visceral metastases.

\section{Conclusions}

Overall, xentuzumab can be safely co-administered with everolimus and exemestane, but the addition of xentuzumab was not associated with improved PFS in the overall population of patients with hormone receptor-positive, HER2-negative locally advanced/MBC. Nonetheless, patients with no visceral involvement or bone-only metastases were identified as subgroups with potential benefit from treatment with Xe1000+Ev10+Ex25, which warrants further investigation. Xentuzumab is currently being investigated in combination with everolimus and exemestane in a double-blind, placebo-controlled, randomised phase II trial $\left(\mathrm{XENERA}^{\mathrm{rm}}-1\right)$ in women with hormone receptor-positive/HER2-negative locally advanced/MBC and non-visceral disease (NCT03659136). The inclusion criteria of XENERA ${ }^{\text {tw }}-1$ also reflect the changing landscape in hormone receptor-positive MBC. Given the time the current trial was conducted (enrolment between 2014 and 2016), there were very limited data on prior treatment with a CDK 4/6 inhibitor. Indeed, only two patients had received prior CDK4/6 inhibitors (Table 1). One patient received prior abemaciclib (January 2015 to July 2015) and one received prior palbociclib (April 2015 to June 2015). In the current trial, both were randomised to Ev10+Ex25 and had PFS of 5.4 and 1.5 months, respectively. Patients enrolled in the XENERA ${ }^{\mathrm{m}}-1$ trial are permitted to have received one prior treatment line with a CDK 4/6 inhibitor, and randomisation will also be stratified based on this.

\section{Supplementary Information}

The online version contains supplementary material available at https://doi. org/10.1186/s13058-020-01382-8.

Additional file 1. : Supplementary material. Supplementary methods, tables and figure

\section{Abbreviations}

LA/MBC: Locally advanced and/or metastatic breast cancer; MTD: Maximum tolerated dose; RP2D: Recommended phase II dose; PFS: Progression-free survival; HER2: Human epidermal growth factor receptor; Al: Aromatase inhibitor; CDK: Cyclin-dependent kinase; OS: Overall survival;

mTOR: Mammalian target of rapamycin; PI3K: Phosphoinositide 3-kinase; 
AKT: Protein kinase B; AEs: Adverse events; IGF: Insulin-like growth factor; MBC: Metastatic breast cancer; QD: Once daily; DMC: Data monitoring committee; RECIST: Response Evaluation Criteria in Solid Tumors; DLT: Doselimiting toxicity; TTP: Time to progression; OR: Objective response; CR: Complete response; PR: Partial response; NCI CTCAE: National Cancer Institute Common Terminology Criteria for Adverse Events; HR: Hazard ratio; Cls: Confidence intervals; ORRs: Objective response rates; DCRs: Disease control rates

\section{Acknowledgements}

Medical writing assistance was provided by Fiona Scott, PhD, of GeoMed, an Ashfield company, part of UDG Healthcare plc, and was supported financially by Boehringer Ingelheim during the preparation of this manuscript.

\section{Authors' contributions}

S-Al, LS, TB, and JCo were involved in the conception and design of the study; M-PS, NM, KSL, SM, JAP-F, DA, AG, AP, TB, and JC collected and/or assembled the data; PS, M-PS, JB, S-Al, Y-SL, PN, AG, AP, GJ, LS, R-ME, TB, DC-LH, JC, and JCo were involved in the data analysis and interpretation. PS, DC-LH, TB, and JC were involved in drafting the manuscript, and all other authors critically revised the manuscript. All authors provided final approval of the manuscript for submission and agree to be accountable for all aspects of the work.

\section{Funding}

This work was supported by Boehringer Ingelheim International GmbH.

\section{Availability of data and materials}

To ensure independent interpretation of clinical study results, Boehringer Ingelheim grants all external authors access to all relevant material, including participant-level clinical study data, and relevant material as needed by them to fulfil their role and obligations as authors under the ICMJE criteria. Furthermore, clinical study documents (e.g. study report, study protocol, statistical analysis plan) and participant clinical study data are available to be shared after publication of the primary manuscript in a peer-reviewed journal and if regulatory activities are complete and other criteria met per the BI Policy on Transparency and Publication of Clinical Study Data: https://trials. boehringer-ingelheim.com/transparency_policy.html

Prior to providing access, documents will be examined and, if necessary, redacted and the data will be de-identified, to protect the personal data of study participants and personnel, and to respect the boundaries of the informed consent of the study participants.

Clinical Study Reports and Related Clinical Documents can be requested via this link: https://trials.boehringer-ingelheim.com/trial_results/clinical_ submission_documents.html

All such requests will be governed by a Document Sharing Agreement. Bona fide, qualified scientific and medical researchers may request access to de-identified, analysable participant clinical study data with corresponding documentation describing the structure and content of the datasets. Upon approval, and governed by a Data Sharing Agreement, data are shared in a secured data-access system for a limited period of 1 year, which may be extended upon request.

Researchers should use https://trials.boehringer-ingelheim.com to request access to study data.

\section{Ethics approval and consent to participate}

The study was performed in accordance with the Declaration of Helsinki and the International Conference on Harmonisation Guideline (ICH) for Good Clinical Practice (GCP). The Independent Ethics Committee (NRES Committee North East, Sunderland, UK) of the coordinating investigator of the trial (Dr Peter Schmid, London, UK) gave a favourable opinion for the study. The IECS and/or IRBs met the requirements of the ICH GCP and local legislation. The Medicines and Healthcare products Regulatory Agency (MHRA) approved the trial on 17 March 2014.

\section{Consent for publication}

Not applicable

\section{Competing interests}

PS declares an advisory council or committee role for Pfizer, AstraZeneca, Novartis, Roche/Genentech, MSD, Boehringer Ingelheim, Bayer, Eisai, Celgene, and Puma; has received honoraria and consulting fees from Pfizer, AstraZeneca, Novartis, Roche/Genentech, Merck, Boehringer Ingelheim, Bayer, Eisai, Celgene, and Puma; and has received grants or research funds for his institution from AstraZeneca, Roche, Genentech, Oncogenex, Novartis, and Astellas. JB declares that his institute received payments from Boehringer Ingelheim for conducting this trial, and his institutes (Karolinska University Hospital and/ or Karolinska Institutet) have received unrestricted research grants to perform academic clinical studies/spin-off studies from Amgen, AstraZeneca, Bayer HealthCare, Merck, Roche, Pfizer, and Sanofi Aventis.

S-Al declares an advisory council or committee role for AstraZeneca, Hanmi, Novartis, MediPacto, Roche, and Pfizer; has received consulting fees from AstraZeneca, Amgen, Hanmi, Novartis, MediPacto, Eisai, Roche, and Pfizer; and has received grants or research funds from AstraZeneca, Roche, and Pfizer. Y-SL declares an advisory council or committee role for Boehringer Ingelheim and Novartis; has received honoraria from Novartis, Pfizer, Roche, Merck Sharp \& Dohme, and Eisai; has received consulting fees from Novartis, Pfizer, Roche, and Merck Sharp; has received grants or research funds from Novartis and Roche, and grants from Merck Sharp \& Dohme; and has received travel grants or lecture fees from Novartis, Pfizer, Roche, Merck Sharp \& Dohme, and Eisai.

NM declares an advisory council or committee role for Eisai, AstraZeneca, Roche, Pfizer, and Novartis.

PN declares an advisory council or committee role and has received consulting fees (with payment to his institution) for Novartis, Pfizer, and Eli Lilly; he has received grants or research funds from Kom op Tegen Kanker Fund. KSL has received consulting fees from Roche, Lilly, and Novartis and has received grants or research funds from Dong-A ST.

JAP-F is a member of a Steering Committee for two trials for AstraZeneca and Karyopharm; has received honoraria from AstraZeneca, Tesaro, Pharmamar, Roche, and Gilead; and has received consulting fees from AstraZeneca, Clovis, Roche, and Tesaro.

DA declares research funding from Boehringer Ingelheim and Roche. AG declares receipt of travel grants from Roche, Novartis, Pfizer, and AstraZeneca.

AP declares advisory council or committee roles for Nanostring Technologies, Roche, Novartis, Pfizer, Oncoloytics Biotech, Amgen, Lilly, MSD, PUMA, and Daiichi Sankyo, Inc, and grants or research funds from Nanostring Technologies, Roche, and Novartis.

GJ declares honoraria from Novartis, Roche, Lilly, Pfizer, Amgen, and BristolMyers Squibb; an advisory role for Novartis, Roche, Amgen, Pfizer, BristolMyers Squibb, Lilly, AstraZeneca, Daiichi Sankyo, and Abbvie; research funding from Roche and Pfizer; and travel, accommodation, or expenses from Novartis, Roche, Pfizer, and Lilly.

LS is employed on behalf of Boehringer Ingelheim.

R-ME and DC-LH are employees of Boehringer Ingelheim.

TB is a former employee of Boehringer Ingelheim and holds a patent for xentuzumab.

JC declares employment and ownership of stock/shares with Oncomark Limited; is on the Board of Directors for Cancer Clinical Research Trust and Cancer Trials Ireland; has received honoraria from Eisai, Amgen, Puma Biotechnology, Seattle Genetics, Boehringer Ingelheim, Pfizer, Vertex Pharmaceuticals, Genomic Health, G1 therapeutics, AstraZeneca, Roche, MSD Oncology, and Pierre Fabre; has a consulting or advisory role with Eisai, Puma Biotechnology, Boehringer Ingelheim, Pfizer, Vertex Pharmaceuticals, Roche, Seattle Genetics, and Novartis; is part of the Speaker's bureau for Pfizer, Eisai, and Genomic Health; has received research funding from Roche, Eisai, Boehringer Ingelheim, and Puma Biotechnology; and has received travel, accommodation, and expenses from MSD, Pfizer, Roche, AstraZeneca, Abbvie, Novartis, and Pierre Fabre.

JCo is a consultant/advisor for Roche, Celgene, Cellestia, AstraZeneca, Biothera Pharmaceutical, Merus, Seattle Genetics, Daiichi Sankyo, Erytech, Athenex, Polyphor, Lilly, Servier, Merck Sharp \& Dohme, GSK, and Leuko; has received honoraria from Roche, Novartis, Celgene, Eisai, Pfizer, Samsung Bioepis, Lilly, Merck Sharp \& Dohme, and Daiichi Sankyo; has received research funding to his institution from Roche, Aria Pharmaceuticals, AstraZeneca, Baxalta, GMBH/Servier Affaires, Bayer HealthCare, Eisai, F Hoffman-La Roche, Guardant Health, Merck Sharp \& Dohme, Pfizer, Piqur Therapeutics, Puma C, Queen Mary University of London, and Seagen; and holds stock, patents, or intellectual property with MedSIR. M-PS and SM have no conflicts of interest to declare. 


\section{Author details}

${ }^{1}$ Centre for Experimental Cancer Medicine, Barts Cancer Institute, Queen Mary University of London, London, UK. ${ }^{2}$ Department of Drug Development and Innovation, Institut Curie, Paris, France. ${ }^{3}$ Department of

Oncology-Pathology, Karolinska Institutet and Breast Cancer Centre, Cancer Theme, Karolinska University Hospital, Stockholm, Sweden. ${ }^{4}$ Department of Internal Medicine, Seoul National University Hospital, Cancer Research Institute, Seoul National University College of Medicine, Seoul, South Korea. ${ }^{5}$ Department of Oncology, National Taiwan University Hospital, Taipei, Taiwan. ${ }^{6}$ Department of Oncology, Ramon y Cajal University Hospital, Madrid, Spain. ${ }^{7}$ Department of Oncology, UZ Leuven, Campus Gasthuisberg, Leuven, Belgium. ${ }^{8}$ Department of Internal Medicine, National Cancer Center, Goyang, South Korea. ${ }^{9}$ Department of Medical Oncology, Hospital Universitario Arnau de Vilanova de Lleida, Lleida, Spain. ${ }^{10}$ Medical Oncology Unit, Hospital Clinico Universitario Valencia, Biomedical Research Institute INCLIVA, CIBERONC, Valencia, Spain. ${ }^{11}$ Department of Medical Oncology, Ninewells Hospital, Tayside Cancer Centre, Dundee, UK. ${ }^{12}$ Department of Medical Oncology, Institut Paoli Calmettes, Aix-Marseille University, CRCM, CNRS, INSE RM, Marseille, France. ${ }^{13}$ Translational Genomics and Targeted Therapeutics in Solid Tumors, IDIBAPS, Hospital Clínic of Barcelona, Barcelona, Spain.

${ }^{14}$ Department of Medical Oncology, Centre Hospitalier Universitaire de Liège, and Liège University, Liège, Belgium. ${ }^{15}$ External statistician on behalf of Boehringer Ingelheim Pharma GmbH \& Co. KG., Staburo GmbH \& Co. KG., Munich, Germany. ${ }^{16}$ Medical Department (Clinical Operations), Boehringer Ingelheim España S.A, Barcelona, Spain. ${ }^{17}$ Medical Department, Boehringer Ingelheim, RCV, Vienna, Austria. ${ }^{18}$ Present Address: Amal Therapeutics SA, Geneva, Switzerland. ${ }^{19}$ Medical Department, Boehringer Ingelheim Taiwan Limited, Taipei, Taiwan. ${ }^{20}$ Present Address: MSD Taiwan, Taipei, Taiwan. ${ }^{21}$ Department of Medical Oncology, St Vincent's University Hospital, Dublin, Ireland. ${ }^{22}$ Breast Cancer Group, Vall d'Hebron Institute of Oncology (VHIO), Barcelona, Spain. ${ }^{23}$ Department of Oncology, IOB Institute of Oncology, Quironsalud Group, Madrid and Barcelona, Spain.

Received: 3 July 2020 Accepted: 9 December 2020

Published online: 15 January 2021

\section{References}

1. Cardoso F, Senkus E, Costa A, Papadopoulos E, Aapro M, André F, et al. 4th ESO-ESMO international consensus guidelines for advanced breast cancer (ABC 4)t. Ann Oncol. 2018;29(8):1634-57.

2. Johnston SR. Enhancing endocrine therapy for hormone receptor-positive advanced breast cancer: cotargeting signaling pathways. J Natl Cancer Inst. 2015;107(10):djv212.

3. Slamon DJ, Neven P, Chia S, Fasching PA, De Laurentiis M, Im S-A, et al. Overall survival with ribociclib plus fulvestrant in advanced breast cancer. N Engl J Med. 2020;382(6):514-24.

4. Sledge GW Jr, Toi M, Neven P, Sohn J, Inoue K, Pivot X, et al. The effect of abemaciclib plus fulvestrant on overall survival in hormone receptorpositive, ERBB2-negative breast cancer that progressed on endocrine therapy-MONARCH 2: a randomized clinical trial. JAMA Oncol. 2019;6(1): $116-24$.

5. Robinson DR, Wu YM, Vats P, Su F, Lonigro RJ, Cao X, et al. Activating ESR1 mutations in hormone-resistant metastatic breast cancer. Nat Genet. 2013; 45(12):1446-51.

6. Baselga J, Campone M, Piccart M, Burris HA 3rd, Rugo HS, Sahmoud T, et al. Everolimus in postmenopausal hormone-receptor-positive advanced breast cancer. N Engl J Med. 2012;366(6):520-9.

7. Piccart M, Hortobagyi GN, Campone M, Pritchard KI, Lebrun F, Ito Y, et al. Everolimus plus exemestane for hormone-receptor-positive, human epidermal growth factor receptor-2-negative advanced breast cancer: overall survival results from BOLERO-2dagger. Ann Oncol. 2014;25(12):2357-62.

8. Cook M, Rabadi LA, Mitri ZI. Everolimus and exemestane for the treatment of metastatic hormone receptor-positive breast cancer patients previously treated with CDK4/6 inhibitor based therapies. J Clin Oncol. 2019;37(15_ suppl):1058.

9. Simpson A, Petnga W, Macaulay VM, Weyer-Czernilofsky U, Bogenrieder T. Insulin-like growth factor (IGF) pathway targeting in cancer: role of the IGF axis and ppportunities for future combination studies. Target Oncol. 2017; 12(5):571-97.

10. Friedbichler K, Hofmann MH, Kroez M, Ostermann E, Lamche HR, KoessI C, et al. Pharmacodynamic and antineoplastic activity of Bl 836845, a fully human IGF ligand-neutralizing antibody, and mechanistic rationale for combination with rapamycin. Mol Cancer Ther. 2014;13(2):399-409.

11. Rieunier G, Wu X, Macaulay VM, Lee AV, Weyer-Czernilofsky U, Bogenrieder T. Bad to the bone: the role of the insulin-like growth factor axis in osseous metastasis. Clin Cancer Res. 2019;25(12):3479-85.

12. LeBedis C, Chen K, Fallavollita L, Boutros T, Brodt P. Peripheral lymph node stromal cells can promote growth and tumorigenicity of breast carcinoma cells through the release of IGF-I and EGF. Int J Cancer. 2002;100(1):2-8.

13. de Bono J, Lin C-C, Chen L-T, Corral J, Michalarea V, Rihawi K, et al. Two first-in-human studies of xentuzumab, a humanised insulin-like growth factor (IGF)-neutralising antibody, in patients with advanced solid tumours. Br J Cancer. 2020;122(9):1324-32.

14. Roberston J, Di Leo A, Fazal M, Lichfield J, Ellis M. Abstract PD5-09: Fulvestrant for hormone receptor-positive advanced breast cancer in patients with visceral vs non-visceral metastases: findings from FALCON, FIRST, and CONFIRM. Cancer Res. 2018;78(4_suppl):PD5-09.

15. Hiraga T, Myoui A, Hashimoto N, Sasaki A, Hata K, Morita Y, et al. Bonederived IGF mediates crosstalk between bone and breast cancer cells in bony metastases. Cancer Res. 2012;72(16):4238-49.

16. Li ZJ, Ying XJ, Chen HL, Ye PJ, Chen ZL, Li G, et al. Insulin-like growth factor1 induces lymphangiogenesis and facilitates lymphatic metastasis in colorectal cancer. World J Gastroenterol. 2013;19(43):7788-94.

17. Morgillo F, De Vita F, Antoniol G, Orditura M, Auriemma PP, Diadema MR, et al. Serum insulin-like growth factor 1 correlates with the risk of nodal metastasis in endocrine-positive breast cancer. Curr Oncol. 2013;20(4):e283-8.

18. Spiliotaki M, Mavroudis D, Kokotsaki M, Vetsika EK, Stoupis I, Matikas A, et al. Expression of insulin-like growth factor-1 receptor in circulating tumor cells of patients with breast cancer is associated with patient outcomes. Mol Oncol. 2018;12(1):21-32.

\section{Publisher's Note}

Springer Nature remains neutral with regard to jurisdictional claims in published maps and institutional affiliations.

\section{Ready to submit your research? Choose BMC and benefit from:}

- fast, convenient online submission

- thorough peer review by experienced researchers in your field

- rapid publication on acceptance

- support for research data, including large and complex data types

- gold Open Access which fosters wider collaboration and increased citations

- maximum visibility for your research: over $100 \mathrm{M}$ website views per year

At BMC, research is always in progress.

Learn more biomedcentral.com/submissions 